\title{
Octreotide long-acting release (LAR) in combination with other therapies for treatment of neuroendocrine neoplasia: a systematic review
}

\author{
Maria Rinzivillo ${ }^{1}$, Ilaria De Felice ${ }^{1}$, Ludovica Magi ${ }^{1}$, Bruno Annibale ${ }^{2}$, Francesco Panzuto ${ }^{1}$ \\ ${ }^{1}$ Digestive Disease Unit, Sant' Andrea University Hospital, ENETS Center of Excellence, Rome, Italy; ${ }^{2}$ Digestive Disease Unit, Sant' Andrea \\ University Hospital, Department of Medical-Surgical Sciences and Translational Medicine, Sapienza University of Rome, ENETS Center of \\ Excellence, Rome, Italy \\ Contributions: (I) Conception and design: M Rinzivillo, F Panzuto; (II) Administrative support: F Panzuto; (III) Provision of study materials or \\ patients: None; (IV) Collection and assembly of data: All authors; (V) Data analysis and interpretation: All authors; (VI) Manuscript writing: All \\ authors; (VII) Final approval of manuscript: All authors. \\ Correspondence to: Francesco Panzuto, MD, PhD. Digestive Disease Unit, Sant' Andrea University Hospital, ENETS Center of Excellence, Via di \\ Grottarossa 1035-39, 00189, Rome, Italy. Email: fpanzuto@ospedalesantandrea.it.
}

Background: In the last decades, the incidence of neuroendocrine neoplasia (NEN) increased from 1 to 5 new diagnoses/100,000 persons/year. The synthetic somatostatin analogues (SSAs) represent the first-choice treatment for both functionally active and inactive gastro-enteric-pancreatic NEN. This systematic review examines the role of octreotide long-acting release (LAR) in combination with other therapies for NEN management.

Methods: Primary outcomes were the disease control rate and the progression free survival (PFS), defined as the time between treatment initiation and progression of disease. Secondary outcomes were overall survival (OS) and safety.

Results: This systematic review identified 13 studies, concerning the use of octreotide LAR in association with other therapies in advanced NENs and included 1,206 patients. Patients were treated with octreotide LAR in combination with other drugs, mainly with everolimus (404 patients, 35\%), but even with Peptide Receptor Radionuclide Therapy, bevacizumab, interferon or fluoride-derivatives. Disease control was observed in $85 \%$ cases with SSAs in combination with other therapies; PFS ranged from 15 to 16.4 months and OS from 25 to 61.9 months. SSAs are very well tolerated drugs, with few side effects which are usually mild, not requiring drug withdrawn.

Conclusions: The review summarizes the effectiveness and available safety data on octreotide LAR in combination with other therapies in patients with NEN and may provide suggestions to address the therapeutic strategy. Further comparative head-to-head studies are needed to understand which is the best combination treatment for patients with progressive NEN after failure of first-line therapy.

Keywords: Neuroendocrine neoplasia (NEN); octreotide; lanreotide; somatostatin analogue; systematic review; everolimus; peptide receptor radionuclide therapy

Submitted Jul 24, 2020. Accepted for publication Dec 07, 2020.

doi: 10.21037/jgo-20-292

View this article at: http://dx.doi.org/10.21037/jgo-20-292

(C) Journal of Gastrointestinal Oncology. All rights reserved. 


\section{Introduction}

Although neuroendocrine neoplasia (NEN) is commonly presented as rare cancer of the neuroendocrine system, their incidence is rising in the last decades, as reported in the epidemiological studies on SEER registries in US that indicated a significant increase in incidence over the last decades (1). The main negative prognostic factors are the primary tumour location [pancreatic NEN (p-NENs) has poor prognosis that small intestine SI-NEN], TNM staging, and the WHO histological classification (2-7). The proliferation index Ki-67 correlates with overall survival (OS) and progression free survival (PFS) in pancreatic NEN, both in advanced and curative phase (7-10). The expression of somatostatin receptor, the speed of spontaneous tumour evolution and patient age can be considered as further prognostic factors.

Overall, NEN are characterized by a relatively long survival. Therefore, they can be managed with sequential treatments, including synthetic somatostatin analogues (SSA) octreotide and lanreotide. These drugs represent the first-choice therapy for both functionally active and inactive GEP-NEN and have been used as comparator for other NEN treatments in many studies (11). SSAs interact with somatostatin receptors, differentially expressed by healthy and tumor cells, and interfere with numerous signal transduction pathways, thus inhibiting adenylate cyclase or regulating ion channels and the catalytic activity of serine/ threonine and tyrosine kinases and phosphatases; all these effects ultimately determine the tumor growth (12). It has been reported that SSAs improved clinical symptoms in many cases, stabilized tumoral growth in $30-50 \%$ of cases, and promoted tumour regression in few cases $(13,14)$. In epidemiological studies on large cohorts, SSAs affected also the survival $(15,16)$.

To date, several therapeutic options have become available to be combined with SSAs in the NEN treatment. Specifically, the somatostatin analogue octreotide has been tested in combination with different compounds, including peptide receptor radionuclide therapy (PRRT), target therapies, anti-angiogenic drugs, and novel molecules.

This systematic review examines the role of octreotide LAR in combination with other therapies in the treatment of patients with advanced NEN, both in terms of effectiveness and safety.

We present this article following the PRISMA reporting checklist (available at: http://dx.doi.org/10.21037/jgo-20-292).

\section{Methods}

\section{Inclusion criteria}

Studies reporting the outcome of octreotide long-acting release (LAR) in combination with other therapies for treatment of patients with NEN were considered. Studies were included regardless of study type, language, publication status, or sample size. Randomized controlled trials (RCT), quasi-randomized controlled trials, and nonrandomized controlled prospective trials were considered for analysis. Retrospective studies and case-control studies and case reports or case series were excluded.

\section{Search strategy}

Medline and Embase were searched until September 2019 using the specific search terms, as detailed in Appendix 1. The methodology followed the Preferred Reporting Items for Systematic Review and Meta-Analyses (PRISMA) statement (17). The bibliography of all papers was studied to pick out further relevant articles, and all titles were reviewed to verify the relevance. Then, we analysed both abstracts and/or full texts of potentially relevant papers were further evaluated. The most recent or more complete publication was considered in presence of duplicates.

\section{Outcome measures}

Primary outcomes were the disease control rate (DCR) and PFS defined as the interval between treatment initiation and progression of disease, e.g., increasing the number or size of the lesions, as determined by imaging studies. Secondary outcome was OS, expressed as the proportion of patients alive after $1,3,5$, or 10 years from the intervention, and/ or as median survival of the group. Mortality and morbidity related to the treatment, and symptomatic relief were also considered.

\section{Data extraction}

Two reviewers (MR and LM) performed search, study selection and data extraction, independently. In case of disagreement on obtained results, a third reviewer (FP) was involved. From each trials we collected the following information: each trial: first author and year of publication, number of patients included in both arms, time from diagnosis to enrolment, reported reason for withdrawal, 


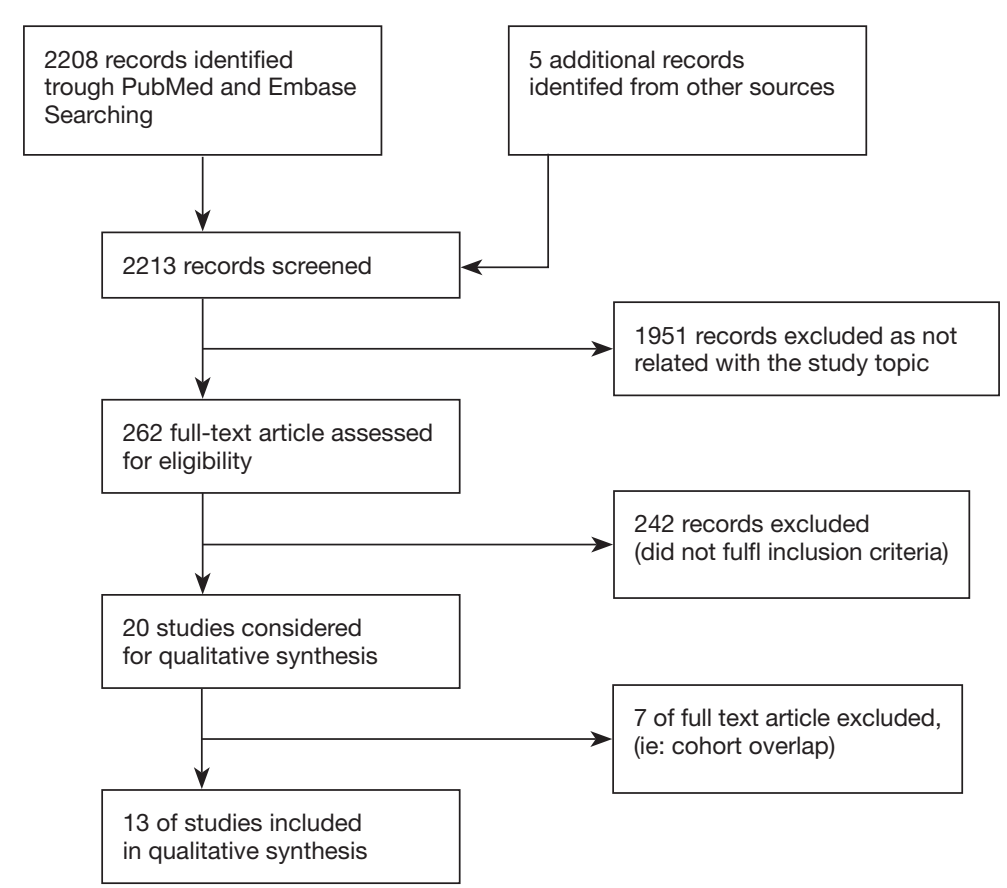

Figure 1 Search results and exclusion criteria, according to PRISMA statement.

tumour primary sites, ki-67/G grading tumour liver burden, treatment (drug name and dosage), hazard ratio (HR) and response rate (RR), complete response (CR), partial response (PR), stable disease (SD) and disease progression (DP) rates, tumour-related death cases, adverse events. We used published data without contacting the authors.

\section{Risk of bias}

Two authors (MR and LM), in parallel, following the instructions reported on the Cochrane Handbook for Systematic Reviews of Intervention (18), determined the study quality. A third author (FP) resolved eventual disagreements. We ascertained also that the RCTs were adequate in terms of sequence generation, allocation concealment, blinding, and completeness of data on outcomes and lacking selective reporting, those studies that had all these characteristics were considered to have a "low or unclear risk of bias".

\section{Statistical analysis}

Considering the high heterogeneity of studies, we presented all relevant data without performing a formal statistical analysis.

\section{Results}

\section{Search result and study selection}

As shown in Figure 1, 2,213 references were identified throughout Medline search.

A total of 1,951 studies were excluded as no related to the topic. The abstracts and/or full text of 262 studies were examined in more detail, and 242 of them were further eliminated, again not addressing the matter, although dealing with NENs. The remnant 20 studies were examined, and 13 papers were included in the review (19-31). Seven papers were excluded due to cohort overlapping or not clearly and explicit results. The agreement for identification of such studies was $100 \%$ between the two reviewers. All these studies described the characteristics of patients with NEN treated with octreotide LAR in combination with other therapies (Table 1). Data from these studies were extracted and qualitatively analysed to highlight suitable evidence.

\section{Study characteristics}

Descriptive characteristics of studies qualitatively analyzed are summarized in Table 1: six were randomized clinical trials (RCTs) and seven were prospective studies; five studies were multicentric. 
Table 1 Characteristics of studies included in the analysis

\begin{tabular}{|c|c|c|c|c|}
\hline First author & Year & $\mathrm{RCT}$ & Octreotide-based therapy & Population \\
\hline Arnold (25) & 2005 & Yes & Octreotide plus interferon & 109 patients with advanced GEP NEN \\
\hline Yao (24) & 2008 & Yes & Octreotide plus everolimus & 60 patients with GEP NEN \\
\hline Brizzi (29) & 2009 & No & Octreotide plus 5-FU & 29 patients with advanced NEN \\
\hline Berrutti (28) & 2014 & No & $\begin{array}{l}\text { Octreotide plus bevacizumab plus } \\
\text { capecitabine }\end{array}$ & 45 patients with advanced NEN \\
\hline Bajetta (21) & 2014 & No & Octreotide plus everolimus & 50 patients with advanced GEP and lung NEN \\
\hline Tippeswamy (22) & 2015 & No & Octreotide plus everolimus & 16 patients with advanced NEN \\
\hline Bendell (27) & 2016 & No & $\begin{array}{l}\text { Octreotide plus bevacizumab plus } \\
\text { pertuzumab }\end{array}$ & 43 patients with advanced NEN \\
\hline Strosberg (19) & 2017 & Yes & Octreotide plus PRRT & 229 patients with patients with advanced midgut NEN \\
\hline Yao (23) & 2017 & Yes & $\begin{array}{l}\text { Octreotide plus INF vs. octreotide } \\
\text { plus bevacizumab }\end{array}$ & 427 patients with advanced NEN \\
\hline Capdevila (30) & 2019 & No & Octreotide plus everolimus & 43 patients with advanced GI NEN \\
\hline
\end{tabular}

Globally, 1,206 patients were treated with octreotide LAR in combination with other therapies, mainly with everolimus (404 patients, $35 \%$ ), as reported in 6 out 13 studies $(20-22,24,26,30)$. In one study, octreotide was used in combination with PRRT (19), whereas in the remaining six studies $(23,25,27-29,31)$ it was administered in combination with bevacizumab or interferon or fluoride-derivatives.

Most patients (98\%) had metastatic disease [this information is not reported in (21) and (25)]; 110 patients had primitive p-NEN and 1,096 non p-NEN, most of them with SI-NENs. Age ranged from 55 years (24) to 66 years (26). In all studies, octreotide dosing was LAR $30 \mathrm{mg}$ every 28 days intra-muscle, except in Dasari et al. in which dose was $20 \mathrm{mg}$ (26) and in the SWOG study where an octreotide $20 \mathrm{mg}$ depot was used (23). In all studies (19-31), patients' grading was G1 or G2 (Ki-67 proliferative index $<20 \%$ ).

\section{Disease progression}

\section{Octreotide + everolimus}

In the studies where octreotide was administrated in combination with everolimus (20-22,24,26,30), the reached
PFS was from 15 months (24) from 16.4 months (20) (expressed as median). In GETNE 1003 Trial (30), PFS was expressed as mean (10.2 months, 95\% CI: 9.3-11.1 months), and the median was not estimable since progression or death were reported in less than half of patients. The efficacy of combination of octreotide with everolimus was tested also in the trial by Bajetta et al., in which the efficacy was not expressed as PFS, but only with time to progression that was calculated in 33.6 months (95\% CI: 18.7-41.2), after a median follow-up of 50 months (21). Tippeswamy did not reported PFS data (22), and Dasari reported a median of PFS in line with previous studies (10 months) (26).

\section{Octreotide + PRRT}

Only a randomized clinical trial had studied the combination therapy between octreotide and PRRT (19) were the estimated 20-month PFS rate was $65.2 \%$ (95\% CI: $50.0-76.8)$ in the ${ }^{177} \mathrm{Lu}$-Dotatate group and $10.8 \%$ (95\% CI, 3.5-23.0) in the control group (19). Median PFS was not determined in the ${ }^{177} \mathrm{Lu}$-Dotatate group and was 8.4 months (95\% CI: 5.8-9.1) in the control group (HR for disease progression or death with ${ }^{177} \mathrm{Lu}$-Dotatate $v s$. control, 0.21; 95\% CI: 0.13-0.33; $\mathrm{P}<0.001)(19)$. 


\section{Octreotide + interferon, bevacizumab and fluoropirimide derivates}

In the studies that evaluated the efficacy of octreotide in combination with interferon, PFS data were not available $(25,31)$. However, in Kölby et al., the combination octreotide and IFN- $\alpha$ significantly reduced the risk of disease progression (HR, 0.28; 95\% CI: 0.16-0.45; $\mathrm{P}=0.008$ ) (31). In the SWOG study, the arm treated with octreotide and interferon (24) had a PFS of 15.4 months (95\% CI: 9.6-18.6) (HR, 0.93; 95\% CI: 0.73-1.18; P=0.55). The efficacy of octreotide in combination with bevacizumab was evaluated in SWOG Study (23), Bendel study (27) and Berruti study (28). The PFS had a value between 6.5 months and 16.6 months (95\% CI: 12.9-19.6) in the bevacizumab arm of SWOG Study (23). In Berruti et al., the combination with octreotide + capecitabine + bevacizumab achieved a median PFS of 14.9 months (95\% CI: 2.4-27.5) in the overall population, 14.3 months (95\% CI: 3.5-25.0) in patients with pancreatic cancer, and 14.3 months $(95 \%$ CI: 0.0-38.6) in patients with intestinal tumor or unknown primary cancer site (28).

\section{Disease control rate}

Generally, as expected, disease control was higher in patients treated with octreotide in combination with other drugs.

\section{Octreotide + everolimus}

The disease control rate in the patient treated with Octreotide plus Everolimus were expressed in all studied analysed, although reported with different outcome. Data were not reported in Dasari et al. (26).

In Pavel et al. (20), the combination of Octreotide and Everolimus reduced the estimated risk for progression by $23 \%$.

When the efficacy of Everolimus in combination with Octreotide was expressed as overall response rate (ORR), the ORR was $20 \%$ and $18 \%$ (in the Radiant- 1 and Bajetta study respectively). In ITMO trial, the clinical benefit was around $92 \%$ (21). Intent-to-treat response rate was $20 \%$ in Radiant-1 (24). Per protocol, there were 13 patients with partial responses (22\%), 42 with stable disease $(70 \%)$, and five patients with progressive disease (8\%) (24).

The efficacy of these combinations has been confirmed by Tippeswamy trials, in which the clinical benefit reaches about the 69\% (22). In GETNE trial, $62.3 \%$ (95\% CI: $48-77 \%)$ of patients had stable disease after 12 months of treatment. The confirmed ORR was $2.3 \%$, and stable disease was $58.1 \%$ (30).

\section{Octreotide + PRRT}

The best disease control rate was obtained with octreotide in combination with PRRT; the highest partial response rate occurred with octreotide in combination with PRRT (19) (17 partial response in PRRT and SSA in combination versus 3 partial response in octreotide high dose alone arm), the objective response rate was $18 \%$ in PRRT + octreotide LAR (19).

\section{Octreotide + interferon, bevacizumab and fluoropirimide derivates}

Concerning the combination with octreotide with bevacizumab and/or with Interferon there are no clear superior or significant clinical benefits were observed.

However, in Arnold et al. (25)—octreotide + interferon, and in the SWOG study (23)—octreotide + bevacizumab + interferon, no advantage was observed using a combined therapy. In Arnold et al., partial tumor regression and stabilization of disease were evaluated at 3, 6 and 12 months, without showing any significant different between arms (partial tumor regression: $2.9 \%$ at 3 months, $1.9 \%$ at 6 months, and $5.7 \%$ at 12 months; stabilization of tumor growth: $44.8 \%$ at 3 months, $27.6 \%$ at 6 months, and $15.2 \%$ at 12 months) (25). In the SWOG study, no significant differences were observed in the primary end point between groups: the median PFS assessed by central review was 16.6 months (95\% CI: $12.9-19.6)$ in the bevacizumab arm and 15.4 months (95\% CI: 9.6-18.6) in the interferon arm (HR, 0.93; 95\% CI: 0.73-1.18; $\mathrm{P}=0.55$ ) (23). Kolby study did no reported these data (31).

Otherwise, the regimen with XELBEVOCT regimen has proven to be effective with OR of $22.2 \%$ (28), as well as in Bendel et al. (27) that reported $16 \%$ of objective responses, while in partial responses were 71 (almost 4\%).

The combination of octreotide with fluoropiridine derivates demonstrated its efficacy in Brizzi trial, where octreotide was alone in combination and in xelbevoct where in combination of 3 drugs (octreotide, bevacizumab, capecitabine). Brizzi reported a disease control rate in $90 \%$ of cases [partial response in $7(24.1 \%)$ and stable disease in $20(69.0 \%)](29)$.

\section{Overall survival}

Median OS ranged from 25 months (26) to 61.9 months (21) for octreotide in combination with other regimens.

\section{Octreotide + everolimus}

Median OS of octreotide in combination with everolimus 
was not reported by Tippeswamy (22).

The final analysis of RADIANT-2 study indicated that median OS after 271 events was 29.2 months (95\% CI: 23.8-35.9) for the everolimus arm and 35.2 months (95\% CI: 30.0-44.7) for the placebo arm (HR 1.17; 95\% CI: $0.92-1.49)(20)$.

Median OS for the study had not been reached in Radiant-1 trial (24). The survival rates calculated by the Kaplan-Meier analysis were $83 \%$ at 1 year, $81 \%$ at 2 years, and $78 \%$ at 3 years.

In the EVERLAR study, median OS was not reached after 24 months [mean 21.1 months (95\% CI: 19.3-22.9)]. The estimated rate of OS after 24 months $71.7 \%$ (95\% CI: 58.2-85.2\%) (30).

In Bajetta (21), after a median follow-up of 277 days, median time to progression and overall survival were not reached. When the same group published a data updated, the median OS was 61.9 months (32).

\section{Octreotide + PRRT}

The survival improvement when octreotide was administered with PRRT was reported by Strosberg et al., in which data were not sufficiently mature to estimate the median OS in each treatment; however, the ad interim analysis indicated an estimated risk of death as $60 \%$, lower in the ${ }^{177} \mathrm{Lu}$-Dotatate group than in the control group (hazard ratio for death in ${ }^{177} \mathrm{Lu}$-Dotatate group versus control, 0.40; $\mathrm{P}=0.004)$ (19).

\section{Octreotide + interferon, bevacizumab and fluoropirimide derivates}

The combinations of octreotide and bevacizumab with or without interferon were reported with dis-homogenous results.

The benefit on OS of Interferon with octreotide was not demonstrated in two trials $(25,31)$. In Arnold et al, patients who received monotherapy achieved a median survival time, calculated from randomization to final evaluation, of 35 months, while in patients who received the combination, the survival lasted 51 months (HR, 1.19; 95\% CI: 0.67-2.13; $\mathrm{P}=0.55$ ) (25). Otherwise, in Kolby's trial, no significant difference in survival was observed between patients treated with octreotide monotherapy or with the combination octreotide and IFN- $\alpha$, (HR, 0.85; 95\% CI: 0.35-2.05, $\mathrm{P}=0.719$ ) (31).

In interferon arm and bevacizumab arm (SWOG study), there was no significant difference in OS (HR, 1.16; 95\% CI: $0.88-1.55 ; \mathrm{P}=0.29)$. The median $\mathrm{OS}$ was 35.2 months (95\% CI, 33.1-42.8) in the bevacizumab arm and was not reached after 36 months of follow-up in the interferon arm. In terms of survival rate, the comparison between among arms indicated $86 \%$ with bevacizumab vs. $84 \%$ with interferon at 12 months, $67 \%$ with bevacizumab vs. $71 \%$ with interferon at 24 months, and $49 \%$ with bevacizumab versus $56 \%$ with interferon at 36 months (23). Bendel et al. reported a median OS of 26.4 months (27).

In Berruti et al., median OS for the overall population and for subgroups with pancreatic or intestinal or unknown primary cancer was not reached (28).

\section{Safety}

Table 2 summarizes safety data. It was not possible to calculate the total amount of adverse events, since they were not clearly reported in all evaluated studies. As an example, in Tippeswamy (22), most common adverse events were reported, but their absolute value not.

\section{Octreotide + everolimus}

In the EVERLAR study, toxicity caused a dose reduction in $31.8 \%$ of patients and suspension in $75 \%$; twenty serious AEs were reported in 18 patients (30).

In the RADIANT-2 study, most adverse events associated with everolimus + octreotide LAR were grade 1 or 2 and were consistent with the known safety profile of these drugs. 40 patients $(19 \%)$ in the everolimus plus octreotide LAR group and 7 (3\%) in the placebo plus octreotide LAR group discontinued the treatment for adverse events. Stomatitis, rash, fatigue, and diarrhea were most commonly reported as drug-related adverse events (20).

\section{Octreotide + PRRT}

Ninety-five percent of patients in the ${ }^{177} \mathrm{Lu}$-Dotatate group had at least one adverse event during the trial. Adverse events related to treatment were reported by 95 patients $(86 \%)$ in the ${ }^{177} \mathrm{Lu}-$ Dotatate group.

The rates of grade 3 or 4 adverse events were similar in the two groups. However, in the ${ }^{177} \mathrm{Lu}$-Dotatate group transient hematological events were reportedspecifically $1 \%$ of patients experienced neutropenia, $2 \%$ thrombocytopenia, and 9\% lymphopenia; renal toxicity was not observed in these patients (19).

\section{Octreotide + interferon, bevacizumab and fluoropirimide derivates}

In Arnold et al., treatment discontinuation due to adverse events and not for tumor progression occurred in 11 out 
Table 2 Adverse events reported in analysed studies

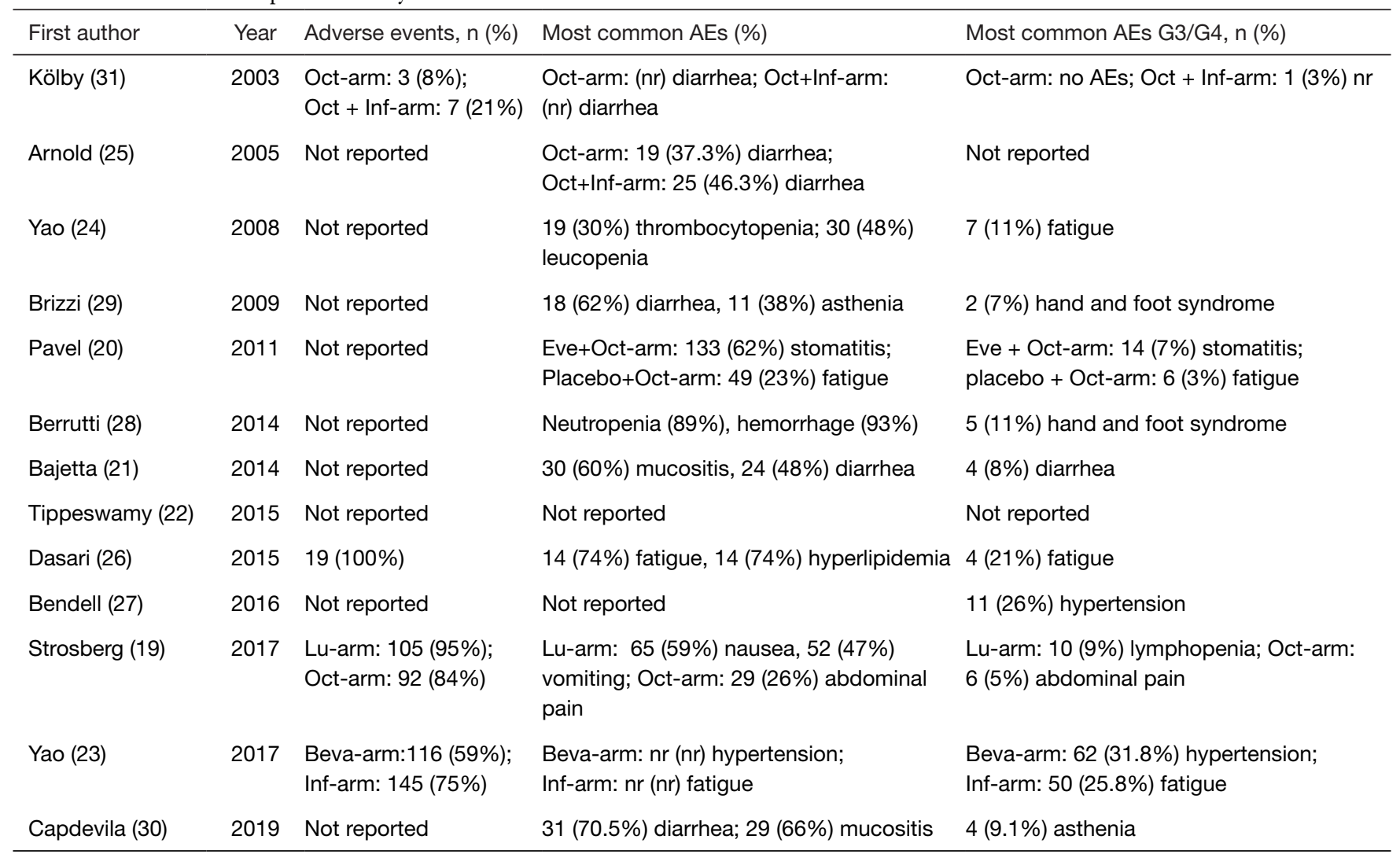

Oct, octreotide; Inf, interferon; Beva, bevacizumab; Lu, ${ }^{177}$ Lu-Dotatate; Eve, everolimus.

of 54 patients who received the combination and in 2 out of 51 patients in monotherapy; more severe adverse events occurred in the combination arm (25). In Berruti et al., the combination of metronomic capecitabine and bevacizumab and concomitant octreotide LAR was well tolerated. Octreotide administration was never delayed nor reduced; the occurrence of proteinuria and hand-foot disease was predictive of response and survival (28).

In Kolby's study the safety profile is not well explained; authors reported that 10 patients-three in group 1 (oct alone) and 7 in group 2 (oct plus INF alpha)-had sideeffects including diarrhea, joint pain, nausea and glucose intolerance (31). One of these side-effects, in a patient in group 2, was reported to be severe.

\section{Quality of life}

The quality of life (QoL) was reported only in two studies $(19,25)$. In Strosberg et al. (19), QoL was defined as the time to QoL worsening that resulted as longer in the ${ }^{177} \mathrm{Lu}-$
Dotatate group than in control arm (28.8 vs. 6.1 months). In Arnold et al., after 3 months of therapy, patients who received the combination reported a reduced global quality of life $(\mathrm{P}<0.003)(25)$.

\section{Discussion}

This systematic review identified 13 studies, concerning the use of octreotide LAR in association with other therapies in advanced NENs. One of the first finding to note is the impressive number of patients $[1,206]$ included in this analysis, given the large amount of available scientific data on octreotide-based combination therapy, that confirm the consolidated role of octreotide in the clinical practice of NEN management. Overall, this study summarizes the positive effect of octreotide, combined with other treatments, in the management of NEN.

Given the heterogeneity of studies in terms of design, combination of treatments, duration of therapy, number of patients included, a conclusive analysis on which is the best 
association for a given kind of NEN patients is not feasible.

However, more solid evidence seems to be available for octreotide plus everolimus or lutetium.

The role of the combination of everolimus and octreotide was stressed for the first time in Bajetta study (21) and in the RADIANT-1 trial (33). Also, in the GETNE trials is clear that the combined therapy showed clinically relevant PFS rate at 12 months of $62 \%$, suggesting a potential benefit for the combination therapy (30).

The hypothesis of a synergic activity between octreotide and other active compounds is supported by several in vivo evidence: SSAs reduce the expression of tumor growth factors, such as insulin-like growth factors and epidermal growth factor, while everolimus can inhibit tumor proliferation, by blocking mTOR signaling and angiogenesis. Therefore, these two drugs seem to synergistically act to block the cellular growth and control the hyper-secretory activity in NENs. The combination of everolimus and SSA showed favorable outcomes, also in patients using metformin (34), as a possible impact of this drug on systemic lipid metabolism, in addition to cellautonomous direct anticancer effects.

As far as the combined use of SSA and PRR is concerned, the schedule including octreotide LAR and ${ }^{177} \mathrm{Lu}-$ Dotatate resulted as significantly efficacious, compared to monotherapy with high-dose octreotide. To date, ${ }^{177} \mathrm{Lu}-$ Dotatate-based PRRT represents the basis for treating welldifferentiated, G1-G2, somatostatin receptors expressing NEN patients who progress after first-line treatment with somatostatin analogues. Basing on the NETTER-1 study protocol (19), octreotide is administered as a maintenance therapy during the interval between each ${ }^{177} \mathrm{Lu}$-Dotatate administration, as well as when PRRT has ended. The therapeutic strategy to maintain cold somatostatin analogues during and after PRRT has been recently proposed to be effective in comparison to PRRT alone by Yordanova et al. (35). In that study, 421 patients with GEP-NENs were included, and patients receiving PRRT alone were compared with patients treated with PRRT and somatostatin analogue (which was octreitide in $77.5 \%$ of cases in the subgroups analysis), which was used also in the maintenance therapy after PRRT stopping. Significantly better PFS and OS rates were observed in patients who received the analogue, confirming that the combination therapy was superior to PRRT alone, again supporting the active additional antiproliferative role of the cold analogue even in the setting of patients who are planned to receive PRRT (35).
Other combinations with octreotide demonstrated as well good clinical outcome, although further data would better specify the potential advantage. The combination octreotide LAR and bevacizumab in the SWOG study achieved a higher response rate with lower toxicity than the treatment with octreotide and interferon; however, the overall efficacy in terms of PFS was similar between treatments (median PFS was 16.6 in bevacizumab arm and 15.4 in interferon arm, HR, 0.93; 95\% CI: 0.73-1.18, $\mathrm{P}=0.55)$ (23).

As far as safety is concerned, it is widely recognized that SSAs are very well tolerated drugs, with few side effects which are usually mild, not requiring drug withdrawn. Among these, gallstones formation is considered as the most serious one. In a recent multi-centre large study performed on 478 NEN patients receiving somatostatin analogues (octreotide in $70 \%$ of them), an incidence for developing gallstones of $27 \%$ was reported, and biliary complications (biliary colic, acute acute cholecystitis, cholangitis, biliary pancreatitis, or obstructive jaundice) occurred in $7.5 \%$ of patients (36). In that study, previous surgery for primary small-bowel NEN was a significant risk factor for developing gallstones, thus defining a specific subgroup of patients at high-risk who should monitor during therapy for this possible side effect, and supporting the recommendation to perform cholecystectomy in those NEN patients who are candidate to surgery for primary small bowel resection. Another uncommon, but possible effect is represented by pancreatic exocrine insufficiency due to inhibition of production and excretion of pancreatic enzymes. This event, which reported in a recent paper with an occurrence of $20 \%$ in patient receiving long-term treatment with SSAs (37), should be considered in this setting. Furthermore, treatment with SSAs may induce hyperglycemia, owing their effect on decreasing insulin resistance (38-40), although data on the real risk for developing diabetes or worsening of pre-existing diabetes in NEN patients receiving these drugs are scanty, owing the lack of prospective studies specifically focusing on this issue.

\section{Conclusions}

Given the rarity and biological heterogeneity of NENs, clinical management of these patients remain a challenge for physicians, requiring a dedicated multidisciplinary team including several specialists. Several questions concerning the optimal therapeutic sequence in these patients are unanswered. Among these, utility to maintain SSAs at time of disease progression is of interest, when a second- 
line treatment is required. Basing on in vivo evidence and considering the analysis performed in the present systematic review, the choice not to withdraw somatostatin analogue in this scenario may be considered safe and effective, irrespective of which second-line treatment is going to be planned.

Octreotide LAR is considered as a milestone in NEN therapy from approximatively two decades, since it is a suitable first-line or maintenance therapy and it can be used in association with second- and third-line options, without additional toxicity in different phases of the disease. A huge amount of scientific data is published on octreotide in combination with other treatments, mainly everolimus and PRRT. Analysing these data through a systematic review approach may be informative for physicians dealing with NENs in the daily clinical practice and may provide useful information on the different therapeutic combinations. Further comparative head-to-head studies are needed to understand which is the best combination treatment for patients with progressive NEN after failure of first-line therapy.

\section{Acknowledgements}

Authors thank Content Ed Net for the editorial support with the helpful contribution of Elisa Sala, PhD, Medical Writer in drafting the test. Authors thank Novartis Farma Italy for funding editorial support.

Funding: None.

\section{Footnote}

Reporting Checklist: The authors have completed the PRISMA reporting checklist. Available at http://dx.doi. org/10.21037/jgo-20-292

Conflicts of Interest: All authors have completed the ICMJE uniform disclosure form (available at: http://dx.doi. org/10.21037/jgo-20-292). Novartis Farma Italy funded the editorial support of the paper. The authors have no other conflicts of interest to declare.

Ethical Statement: The authors are accountable for all aspects of the work in ensuring that questions related to the accuracy or integrity of any part of the work are appropriately investigated and resolved.

Open Access Statement: This is an Open Access article distributed in accordance with the Creative Commons Attribution-NonCommercial-NoDerivs 4.0 International License (CC BY-NC-ND 4.0), which permits the noncommercial replication and distribution of the article with the strict proviso that no changes or edits are made and the original work is properly cited (including links to both the formal publication through the relevant DOI and the license). See: https://creativecommons.org/licenses/by-nc-nd/4.0/.

\section{References}

1. Dasari A, Shen C, Halperin D, et al. Trends in the incidence, prevalence, and survival outcomes inpatients with neuroendocrine tumors in the United States. JAMA Oncol 2017;3:1335-42.

2. Ramage JK, Ahmed A, Ardill J, et al. Guidelines for the management of gastroenteropancreatic neuroendocrine (including carcinoid) tumours (NETs). Gut 2012;61:6-32.

3. Klimstra DS, Kloppell G, La Rosa S, et al. Classification of neuroendocrine neoplasms of the digestive system. In: WHO Classification of Tumours Editorial Board. editor. WHO Classification of Tumours: Digestive System Tumours, 5th ed. Lyon: International Agency for Research on Cancer, 2019; 16.

4. Boudreaux JP, Klimstra DS, Hassan MM, et al. The NANETS consensus guideline for the diagnosis and management of neuroendocrine tumors: well-differentiated neuroendocrine tumors of the Jejunum, Ileum, Appendix, and Cecum. Pancreas 2010;39:753-66.

5. Panzuto F, Boninsegna L, Fazio N, et al. Metastatic and locally advanced pancreatic endocrine carcinomas: Analysis of factors associated with disease progression. J Clin Oncol 2011;29:2372-7.

6. Panzuto F, Merola E, Rinzivillo M, et al. Advanced digestive neuroendocrine tumors: Metastatic pattern is an independent factor affecting clinical outcome. Pancreas 2014;43:212-8.

7. Panzuto F, Campana D, Fazio N, et al. Risk factors for disease progression in advanced jejunoileal neuroendocrine tumors. Neuroendocrinology 2012;96:32-40.

8. Rindi G, Falconi M, Klersy C, et al. TNM staging of neoplasms of the endocrine pancreas: results from a large international cohort study. J Natl Cancer Inst 2012;104:764-77.

9. Rindi G, Kloppel G, Alhman H, et al. TNM staging of foregut (neuro)endocrine tumors: a consensus proposal including a grading system. Virchows Arch 2006;449:395-401.

10. Rindi, G, Klersy, C, Albarello L, et al. Competitive 
Testing of the WHO 2010 versus the WHO 2017 Grading of Pancreatic Neuroendocrine Neoplasms: Data from a Large International Cohort Study. Neuroendocrinology 2018;107:375-86.

11. Bousquet C, Puente E, Buscail L, et al. Antiproliferative effect of somatostatin and analogs. Chemotherapy 2001;47:30-9.

12. Oberg K, Kvols L, Caplin M et al. Consensus report on the use of somatostatin analogs for the management of neuroendocrine tumors of the gastroenteropancreatic system. Ann Oncol 2004;15:966-73.

13. Panzuto F, Di Fonzo M, Iannicelli E, et al. Long-term clinical outcome of somatostatin analogues for treatment of progressive, metastatic, well-differentiated entero-pancreatic endocrine carcinoma. Ann Oncol 2006;17:461-6.

14. Strosberg J, Gardner N, Kvols L. Survival and Prognostic Factor Analysis of 146 Metastatic Neuroendocrine Tumors of the Mid-Gut. Neuroendocrinology 2009;89:471-6.

15. Yao JC, Hassan M, Phan A, et al. One hundred years after 'carcinoid': epidemiology of and prognostic factors for neuroendocrine tumors in 35,825 cases in the United States. J Clin Oncol 2008;26:3063-72.

16. Modlin IM, Pavel M, Kidd M, Gustafsson BI. Review article: somatostatin analogues in the treatment of gastroenteropancreatic neuroendocrine (carcinoid) tumours. Aliment Pharmacol Ther 2010;31:169-88.

17. Moher D, Liberati A, Tetzlaff J, et al. Preferred reporting items for systematic reviews and meta-analyses: the PRISMA statement. PLoS Med 2009;6:e1000097.

18. Higgins JP, Green S. Eds. Cochrane Handbook for Systematic Reviews of Interventions Version 5.1.0 (updated March 2011). The Cochrane Collaboration, 2011. Available online: www.cochrane-handbook.org

19. Strosberg J, El-Haddad G, Wolin E, et al. Phase 3 Trial of 177Lu-Dotatate for Midgut Neuroendocrine Tumors. N Engl J Med 2017;376:125-35.

20. Pavel ME, Hainsworth JD, Baudin E, et al. Everolimus plus octreotide long-acting repeatable for the treatment of advanced neuroendocrine tumours associated with carcinoid syndrome (RADIANT-2): a randomised, placebocontrolled, phase 3 study. Lancet 2011;378:2005-12.

21. Bajetta E, Catena L, Fazio N, et al. Everolimus in Combination With Octreotide Long-Acting Repeatable in a First-Line Setting for Patients With Neuroendocrine Tumors: An ITMO Group Study. Cancer 2014;120:2457-63.

22. Tippeswamy R, Patil S, Sateesh CT, et al. Everolimus plus octreotide long-acting repeatable in advanced neuroendocrine tumors in the routine tertiary cancer care setting: An Indian experience. Indian J Cancer 2015;52:359-62.

23. Yao JC, Guthrie KA, Moran C, et al. Phase III Prospective Randomized Comparison Trial of Depot Octreotide Plus Interferon Alfa-2b Versus Depot Octreotide Plus Bevacizumab in Patients With Advanced Carcinoid Tumors: SWOG S0518. J Clin Oncol 2017;35:1695-703.

24. Yao JC, Phan AT, Chang DZ, et al. Efficacy of RAD001 (everolimus) and octreotide LAR in advanced low- to intermediate-grade neuroendocrine tumors: results of a phase II study. J Clin Oncol 2008;26:4311-8.

25. Arnold R, Rinke A, Klose KJ, et al. Octreotide versus octreotide plus interferon-alpha in endocrine gastroenteropancreatic tumors: a randomized trial. Clin Gastroenterol Hepatol 2005;3:761-71.

26. Dasari A, Phan A, Gupta S, et al. Phase I study of the anti-IGF1R antibody cixutumumab with everolimus and octreotide in advanced well-differentiated neuroendocrine tumors. Endocr Relat Cancer 2015;22:431-41.

27. Bendell JC, Zakari A, Lang E, et al. A Phase II Study of the Combination of Bevacizumab, Pertuzumab, and Octreotide LAR for Patients with Advanced Neuroendocrine Cancers. Cancer Invest 2016;34:213-9.

28. Berruti A, Fazio N, Ferrero A, et al. Bevacizumab plus octreotide and metronomic capecitabine in patients with metastatic well-to-moderately differentiated neuroendocrine tumors: the XELBEVOCT study. BMC Cancer 2014;14:184.

29. Brizzi MP, Berruti A, Ferrero A, et al. Continuous 5-fluorouracil infusion plus long acting octreotide in advanced well-differentiated neuroendocrine carcinomas. A phase II trial of the Piemonte oncology network. BMC Cancer 2009;9:388.

30. Capdevila J, Teulé A, Barriuso J, et al. Phase II Study of Everolimus and Octreotide LAR in Patients with Nonfunctioning Gastrointestinal Neuroendocrine Tumors: The GETNE1003_EVERLAR Study. Oncologist 2019;24:38-46.

31. Kölby L, Persson G, Franzén S, Ahrén B. Randomized clinical trial of the effect of interferon alpha on survival in patients with disseminated midgut carcinoid tumours. Br J Surg 2003;90:687-93.

32. Bajetta E, Catena L, Pusceddu S, et al. Everolimus in Combination with Octreotide Long-Acting Repeatable in a First-Line Setting for Patients with Neuroendocrine Tumors: A 5-Year Update. Neuroendocrinology 2018;106:307-11.

33. Yao JC, Lombard-Bohas C, Baudin E, et al. Daily oral 
everolimus activity in patients with metastatic pancreatic neuroendocrine tumors after failure of cytotoxic chemotherapy: a phase II trial. J Clin Oncol 2010;28:69-76.

34. Pusceddu S, Vernieri C, Di Maio M, et al. Metformin Use Is Associated With Longer Progression-Free Survival of Patients With Diabetes and Pancreatic Neuroendocrine Tumors Receiving Everolimus and/or Somatostatin Analogues. Gastroenterology 2018;155:479-489.e7.

35. Yordanova A, Wicharz MM, Mayer K, et al. The Role of Adding Somatostatin Analogues to Peptide Receptor Radionuclide Therapy as a Combination and Maintenance Therapy. Clin Cancer Res 2018;24:4672-9.

36. Brighi N, Panzuto F, Modica R, et al. Biliary Stone Disease in Patients with Neuroendocrine Tumors Treated with Somatostatin Analogs: A Multicenter Study. Oncologist 2020;25:259-65.

Cite this article as: Rinzivillo M, De Felice I, Magi L, Annibale B, Panzuto F. Octreotide LAR in combination with other therapies for treatment of neuroendocrine neoplasia: a systematic review. J Gastrointest Oncol 2021;12(2):845-855. doi: 10.21037/jgo-20-292
37. Rinzivillo M, De Felice I, Magi L, et al. Occurrence of exocrine pancreatic insufficiency in patients with advanced neuroendocrine tumors treated with somatostatin analogs. Pancreatology 2020;20:875-9.

38. Magi L, Mazzuca F, Rinzivillo M, et al. Multidisciplinary Management of Neuroendocrine Neoplasia: A RealWorld Experience from a Referral Center. J Clin Med 2019;8:910.

39. Ronchi C, Epaminonda P, Cappiello V, Beck-Peccoz P, Arosio M. Effects of two different somatostatin analogs on glucose tolerance in acromegaly. J Endocrinol Invest 2002;25:502-7.

40. Izzedine H, Launay-Vacher V, Deybach C, et al. Druginduced diabetes mellitus. Expert Opin Drug Saf 2005;4:1097-109. 


\section{Supplementary}

\section{Appendix 1 Search strategy}

\section{Final EMBASE:}

('neuroendocrine tumour'/exp OR 'neuroendocrine tumour' OR 'carcinoid' OR 'bronchus carcinoid' OR 'pancreas islet cell tumour') AND ('octreotide' OR 'somatostatin derivative')) AND ('everolimus' OR 'mammalian target of rapamycin inhibitor' OR 'mtor signaling' OR 'sunitinib' OR 'vasculotropin receptor 2' OR 'oxodotreotide Lu 177' OR 'dotatoc' OR 'dotatoc Lu 177' OR ‘dotatoc y 90’ OR 'dotatate y 90' OR 'radioisotope therapy' OR 'peptide receptor radionuclide therapy') AND (English)/lim

\section{PUBMED:}

((“Neuroendocrine Tumours”(Mesh) OR "Carcinoid Tumour"(Mesh) OR "Gastro-enteropancreatic neuroendocrine tumour" (Supplementary Concept) OR "Carcinoid Tumours, Intestinal" (Supplementary Concept) OR "Non-functioning pancreatic endocrine tumour" (Supplementary Concept)) AND ("Octreotide"(Mesh)) AND ("Everolimus"(Mesh) OR "sunitinib" (Supplementary Concept) OR "(177lutetiumDOTA $(\mathrm{O})$ Tyr3) octreotate" (Supplementary Concept) OR "Immunoconjugates"(Mesh) OR "90Y-octreotide, DOTATyr(3)-” (Supplementary Concept))) 